PAPER • OPEN ACCESS

\title{
Quantum measurement of space-time events
}

To cite this article: Dorje C Brody and Lane P Hughston 2021 J. Phys. A: Math. Theor. 54235304

View the article online for updates and enhancements.

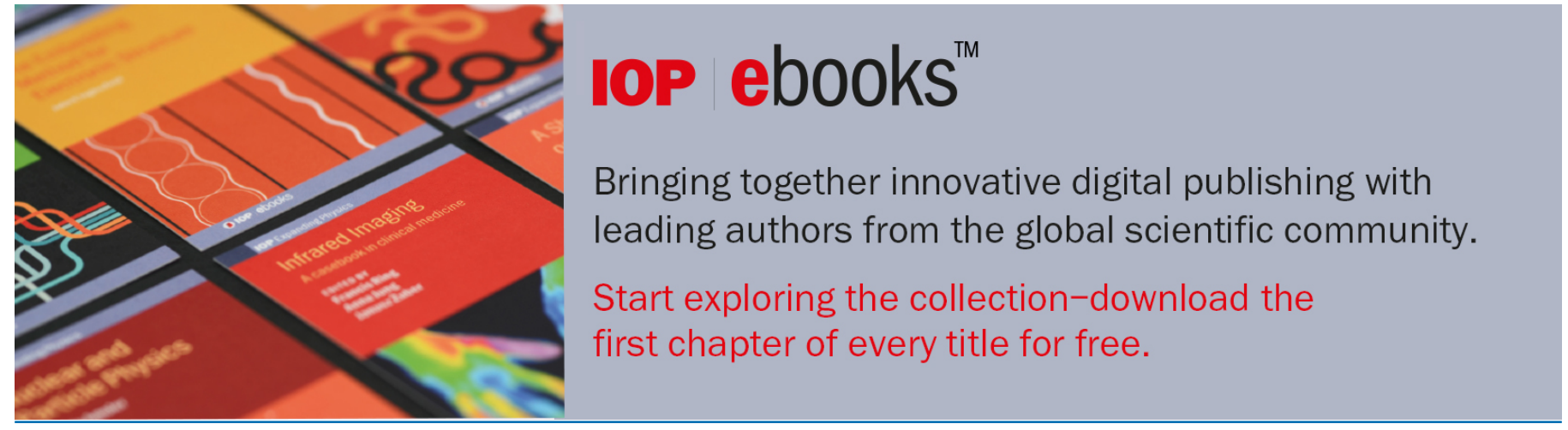

This content was downloaded from IP address 86.184 .59 .98 on $28 / 05 / 2021$ at 22:05 


\title{
Quantum measurement of space-time events
}

\author{
Dorje C Brody $^{1,2, *(1)}$ and Lane P Hughston ${ }^{3}$ (i) \\ 1 Department of Mathematics, University of Surrey, Guildford GU2 7XH, United \\ Kingdom \\ 2 St Petersburg National Research University of Information Technologies, \\ Mechanics and Optics, St Petersburg 197101, Russia \\ ${ }^{3}$ Department of Computing, Goldsmiths University of London, New Cross, London \\ SE14 6NW, United Kingdom
}

E-mail: d.brody@ surrey.ac.uk

Received 23 December 2020, revised 1 April 2021

Accepted for publication 22 April 2021

Published 28 May 2021

\section{Abstract}

The phase space of a relativistic system can be identified with the future tube of complexified Minkowski space. As well as a complex structure and a symplectic structure, the future tube, seen as an eight-dimensional real manifold, is endowed with a natural positive-definite Riemannian metric that accommodates the underlying geometry of the indefinite Minkowski space metric, together with its symmetry group. A unitary representation of the 15-parameter group of conformal transformations can then be constructed that acts upon the Hilbert space of square-integrable holomorphic functions on the future tube. These structures are enough to allow one to put forward a quantum theory of phasespace events. In particular, a theory of quantum measurement can be formulated in a relativistic setting, based on the use of positive operator valued measures, for the detection of phase-space events, hence allowing one to assign probabilities to the outcomes of joint space-time and four-momentum measurements in a manifestly covariant framework. This leads to a localization theorem for phase-space events in relativistic quantum theory, determined by the associated Compton wavelength.

Keywords: relativistic quantum theory, Bergman kernel, future tube, complex Minkowski space, positive operator-valued measures

\footnotetext{
*Author to whom any correspondence should be addressed.
}

Original content from this work may be used under the terms of the Creative Commons Attribution 4.0 licence. Any further distribution of this work must maintain attribution to the author(s) and the title of the work, journal citation and DOI. 


\section{Introduction}

Starting with the pioneering work of Dirac [1], investigations of the Hamiltonian formulation of space-time physics have been pursued by numerous authors. One of the motivations behind such analysis has been that the mathematical structures of phase-space formalisms can be highly amenable to a quantum-mechanical description. The naive formulation of a relativistic phase space as a kind of doubled-up Minkowski space with four position coordinates and four momentum coordinates, while feasible in the classical theory, is not satisfactory as a basis for relativistic quantum theory. Here we propose an alternative approach in which the future tube of complexified Minkowski space is taken to be the phase space of a relativistic system. Remarkably, this phase space comes naturally equipped with both the symplectic structure and the compatible Riemannian structure needed for the development of a fully covariant relativistic quantum theory.

Let us write $\mathbb{M}$ for Minkowski space, by which we mean $\mathbb{R}^{4}$ equipped with the usual flat space-time metric $g_{a b}$ with signature $(+,-,-,-)$. For the positions of points $x, y \in \mathbb{M}$ relative to an origin in $\mathbb{M}$ we write $x^{a}$ and $y^{a}$, where $a, b=0,1,2,3$. We say that $x$ and $y$ are time-like, space-like, or null separated according to whether $g_{a b}\left(x^{a}-y^{a}\right)\left(x^{b}-y^{b}\right)$ is positive, negative, or zero. In the time-like and null cases, the separation vector $v^{a}=x^{a}-y^{a}$ is said to be futurepointing or past-pointing according to whether $v^{0}$ is positive or negative. Then by complex Minkowski space $\mathbb{C M}$ we mean $\mathbb{C}^{4}$ equipped with the same metric tensor. The so-called future tube $\Gamma$ is the open submanifold of $\mathbb{C M}$ consisting of points that are of the form $z^{a}=x^{a}-\mathrm{i} r^{a}$, where $r^{a}$ is time-like and future pointing. Thus for all $z^{a} \in \Gamma$ it holds that $g_{a b} r^{a} r^{b}>0$ with $r^{0}>0$.

The future tube plays an important role in rigorous treatments of quantum field theory. In particular, the Wightman functions - given by vacuum expectations of field operators-are analytic in $\Gamma$, and one can reconstruct the field theory from the data of these expectation values [2-5]. The future tube contains no real space-time points; however, the so-called extended future tube, consisting of points attainable by the action of the complex Lorentz group on $\Gamma$, contains real points, called Jost points. One can then recover the field theory from the values of the Wightman functions at Jost points [6]. Complexified Minkowski space also plays an important role in the Penrose twistor program [7-9], as does the future tube. In twistor theory, the complex projective space $\mathbb{C P}^{3}$ is divided into two parts, the upper and lower half of $\mathbb{C P}^{3}$, separated by a five real dimensional hypersurface $\mathbb{N}^{5}$ of null twistors. The points of $\mathbb{C M}$ correspond to complex projective lines in $\mathbb{C P}^{3}$. The points of $\Gamma$ correspond to lines that lie entirely in the top half of $\mathbb{C P}^{3}$.

In both twistor theory and quantum field theory, the complexification of Minkowski space, natural as it may be, is introduced primarily to enable one to exploit the tools of complex analysis in relation to the positive frequency condition on fields; and there is no direct physical significance attached as such to the imaginary components of complex space-time points. Some form of reality condition has to be brought into play to make the link to the physical 'real' spacetime.

From the view of the complex formulation of classical mechanics [10], it is natural to ask whether the imaginary part of a point in $\Gamma$ is related to the four momentum of a relativistic system. In what follows we offer an affirmative answer to this question. This, in turn, allows us to construct a Hilbert space of quantum states over the space-time phase space. The conformal transformations of the underlying space-time phase space can then be represented explicitly in terms of unitary operators acting on quantum states. We then formulate a measurement postulate for the detection of the phase-space location of a relativistic event by identifying the probability law for measurement outcomes along with an appropriate post-measurement 
transformation rule for the states. It is shown, in particular, that when the measurement outcome yields a phase-space point, the state transforms into a coherent state of the conformal group, centered at that point. The fact that coherent states are the most localized states in the Hilbert space then leads to a localization bound which shows for systems of short Compton wavelength that when an event is detected to have occurred at a specific phase-space point, the resulting state will be highly localized in phase space.

The problem of relativistic quantum measurement has been investigated by many authors (see e.g. [11-14] and references cited therein). It is often the case, however, that the measurement postulates of nonrelativistic quantum theory are used in a relativistic setup to deduce implications of the postulates, which is unsatisfactory, for what is required is a measurement postulate in a relativistic setup, as we propose here.

\section{Relativistic mechanics}

We begin by reviewing the cotangent bundle approach to relativistic mechanics [15-18]. The phase space is taken to be the cotangent bundle of Minkowski space, where the cotangent vectors in the fibre over a point in Minkowski space are identified with the momentum fourvectors that the particle might possess. The bundle is an eight-dimensional manifold $T^{*} \mathbb{M}$, with base coordinates $x^{a}$ and fibre coordinates $p_{a}$. We form the canonical one-form $\theta=p_{a} \mathrm{~d} x^{a}$ on $T^{*} \mathbb{M}$ along with its exterior derivative, the associated symplectic form $\omega=\mathrm{d} p_{a} \wedge \mathrm{d} x^{a}$. Given a smooth function $H: T^{*} \mathbb{M} \rightarrow \mathbb{R}$ we then write Hamilton's equations for a dynamical trajectory

$$
s \in \mathbb{R}^{+} \mapsto\left\{x^{a}(s), p_{a}(s)\right\} \in T^{*} \mathbb{M}
$$

in the form

$$
\frac{\mathrm{d} x^{a}}{\mathrm{~d} s}=\frac{\partial H}{\partial p_{a}} \quad \text { and } \quad \frac{\mathrm{d} p_{a}}{\mathrm{~d} s}=-\frac{\partial H}{\partial x^{a}}
$$

and we call $H\left(x^{a}, p_{a}\right)$ the Hamiltonian function. As before, we let $g_{a b}=\operatorname{diag}(+1,-1,-1,-1)$ be the metric on the base space $\mathbb{M}$, which we can use to raise and lower indices on the fibre elements as well. Then we can write $\left(x^{a}, p^{a}\right)=\left(x^{a}, g^{a b} p_{b}\right)$ and put Hamilton's equations in the more symmetrical form

$$
\frac{\mathrm{d} x^{a}}{\mathrm{~d} s}=g^{a b} \frac{\partial H}{\partial p^{b}} \quad \text { and } \quad \frac{\mathrm{d} p^{a}}{\mathrm{~d} s}=-g^{a b} \frac{\partial H}{\partial x^{b}} .
$$

The cotangent-bundle approach works well for characterizing the dynamics of typical mechanical systems in space-time. To convince oneself it suffices to explore a few examples.

Example 1: free particle. The Hamiltonian is taken to be

$$
H=\left[g_{a b} p^{a} p^{b}\right]^{1 / 2}
$$

Then $H$ will be a constant of the motion which we identify as the mass $m$. The phase space is foliated by surfaces of constant $H$, and as an initial condition we choose $\left\{x^{a}(0), p_{a}(0)\right\}$ to lie on the surface $H=m$. Hamilton's equation (3) imply

$$
m \dot{x}^{a}=p^{a}, \quad \dot{p}^{a}=0 .
$$

The phase-space trajectory is given by

$$
x^{a}(s)=x^{a}(0)+s m^{-1} p_{a}(0), \quad p_{a}(s)=p_{a}(0),
$$


corresponding to a geodesic motion in Minkowski space subject to the specified initial conditions.

Example 2: charged particle in an electromagnetic field. Let the charge be $q$ and write $A^{a}(x)$ for the electromagnetic four-potential. With the familiar minimal coupling, we extend the previous example by taking the Hamiltonian to be of the form

$$
H=\left[g_{a b}\left(p^{a}-q A^{a}\right)\left(p^{b}-q A^{b}\right)\right]^{1 / 2} .
$$

We foliate the phase space with surfaces of constant $H$, identifying the value of $H$ with the mass of the particle. Hamilton's equations give

$$
m \dot{x}^{a}=\left(p^{a}-q A^{a}\right), \quad \dot{p}^{a}=q \dot{x}_{c} \nabla^{a} A^{c},
$$

where $\nabla_{a}=\partial / \partial x^{a}$. Further differentiation leads to the Lorentz force law

$$
m \ddot{x}^{a}=q F^{a b} \dot{x}_{b}, \quad F^{a b}=\nabla^{a} A^{b}-\nabla^{b} A^{a} .
$$

Example 3: relativistic two-body problem with a force of mutual attraction. Let us write $x^{a}$, $y^{a}, X^{a}, Y^{a}$ for the space-time positions and momenta of the two particles, setting

$$
q^{a}=\frac{1}{2}\left(x^{a}-y^{a}\right)
$$

along with

$$
P^{a}=X^{a}+Y^{a}, \quad Q^{a}=X^{a}-Y^{a} .
$$

To model a central force we project $q^{a}$ onto the space-like hypersurface orthogonal to the total momentum $P^{a}$ to measure the separation of the two particles. Since $P^{a}$ is time-like, the resulting 'internal' coordinate $\xi^{a}$ defined by

$$
\xi^{a}=q^{a}-\frac{q_{c} P^{c}}{P_{c} P^{c}} P^{a}
$$

is space-like. Thus $\xi_{a} \xi^{a} \leqslant 0$ and for the potential we set

$$
V\left(\xi^{a}\right)=\Phi\left(-\xi_{a} \xi^{a}\right)
$$

for some function $\Phi: \mathbb{R}^{+} \backslash\{0\} \rightarrow \mathbb{R}$ in $\mathrm{C}^{1}(0, \infty)$. For example, for a harmonic oscillator we set $\Phi(u)=k u$ where $k \in \mathbb{R}^{+}$. For a Coulomb potential set $\Phi(u)=-e_{1} e_{2} u^{-1 / 2}$, where $e_{1}, e_{2}$ are the charges of the particles. For a gravitational potential, set $\Phi(u)=G m_{1} m_{2} u^{-1 / 2}$, and so on. Consider now a pair of particles interacting via the potential $V$. We write

$$
X^{2}=m_{1}^{2}+V, \quad Y^{2}=m_{2}^{2}+V,
$$

where $X^{2}=X_{a} X^{a}$ and $Y^{2}=Y_{a} Y^{a}$, and $m_{1}, m_{2}$ are the rest masses. These conditions imply

$$
\frac{1}{2}\left(P^{2}+Q^{2}\right)-2 V=m_{1}^{2}+m_{2}^{2}, \quad P_{c} Q^{c}=m_{1}^{2}-m_{2}^{2} .
$$

Hence for the Hamiltonian we set

$$
H=\left(\frac{1}{2}\left(P^{2}+Q^{2}\right)-2 V\right)^{1 / 2} \text {. }
$$


Since $H$ will be a constant of the motion, we choose the initial conditions so that $\left\{r^{a}(0), q^{a}(0)\right\}$ lies on the surface

$$
H=\left[m_{1}^{2}+m_{2}^{2}\right]^{1 / 2} .
$$

Hamilton's equations show that $P_{c} Q^{c}$ is also a constant of the motion, so we set

$$
P_{c} Q^{c}=m_{1}^{2}-m_{2}^{2},
$$

thus fixing the two masses. A calculation then shows that

$$
\frac{\mathrm{d}^{2} \xi^{a}}{\mathrm{~d} s^{2}}=-\frac{1}{m_{1}^{2}+m_{2}^{2}} \Phi^{\prime}\left(-\xi_{c} \xi^{c}\right) \xi^{a},
$$

where $\Phi^{\prime}(u)=\mathrm{d} \Phi(u) / \mathrm{d} u$. Since the right side is a function of $\xi^{a}$, we can solve for $\xi^{a}(s)$, which in turn allows us to determine the phase-space trajectory. For example, in the case of an oscillator, we have $\Phi^{\prime}=k$, so we obtain

$$
\xi^{a}(s)=\alpha^{a} \cos (\omega s)+\beta^{a} \sin (\omega s),
$$

where $\alpha^{a}, \beta^{a}$ are constant spacelike vectors such that $\alpha^{a}=\xi^{a}(0)$ and $\omega \beta^{a}=\dot{\xi}^{a}(0)$, with $\omega^{2}=$ $k /\left(m_{1}^{2}+m_{2}^{2}\right)$.

Despite the merits of these examples, there are limitations to the effectiveness of the cotangent bundle approach as a foundation for the theory of relativistic dynamics. For a start, there is no intrinsic mechanism to prevent the momentum from becoming space-like or past-pointing. This problem can be avoided in specific examples, such as the ones above, but it is undesirable that one should have to manage the situation on an ad hoc basis. The cotangent bundle approach also poses problems when we look at field theories, since the cotangent bundle does not admit a natural complex structure. In particular, there is no general recipe for combining position and momentum in a linear way, allowing one to write complex expressions of the form $x^{a}+\mathrm{i} p^{a}$. Such variables arise in the quantization of oscillators, but in that case there is a dimensional constant that allows one to modify the expressions to produce terms of the same dimensionality. If the constants of nature at ones disposal are the speed of light and Planck's constant, then one cannot convert a quantity with units of momentum to one with units of length. Further, the interpretation of the parameter $s$ as a proper time in the cotangent bundle approach is ambiguous when many particles are involved.

What is the best way forward? Many authors have considered the problems arising with relativistic phase spaces, both for classical theories and quantum theories [19-23]. Our approach incorporates ideas drawn from all of these, and from geometric quantum mechanics as well [24-30]. We also look closely at the role of probability in the course of our development of a relativistic theory of quantum measurement based on the geometry of the future tube.

\section{Back to the future tube}

That there is an appropriate map from the cotangent bundle to the future tube is not immediately apparent, but a dimensional argument will lead the way. In order for us to be able to regard $x^{a}-\mathrm{i} r^{a}$ as a complex phase-space variable in a relativistic context we shall require $r^{a}$ to have units of inverse momentum. Then if we multiply $r^{a}$ by Planck's constant we obtain a vector with units of position that can be combined with $x^{a}$. Specifically, we consider the Kelvin inversion

$$
r^{a}=\hbar p^{a} /\left(p_{c} p^{c}\right), \quad p^{a}=\hbar r^{a} /\left(r^{a} r_{a}\right)
$$


This transformation maps the cone of time-like future-pointing Minkowski space vectors into itself. Thus we have

$$
\hbar g^{a b} \frac{\partial}{\partial p^{b}}=r_{c} r^{c}\left(g^{a b}-\frac{2 r^{a} r^{b}}{r_{c} r^{c}}\right) \frac{\partial}{\partial r^{b}}
$$

and

$$
\frac{1}{\hbar} g_{a b} \frac{\mathrm{d} p^{a}}{\mathrm{~d} s}=\frac{1}{r_{c} r^{c}}\left(g_{a b}-\frac{2 r_{a} r_{b}}{r_{c} r^{c}}\right) \frac{\mathrm{d} r^{a}}{\mathrm{~d} s}
$$

Now define a symmetric tensor $h_{a b}$ with inverse $k^{a b}$ by setting

$$
h_{a b}=-\frac{1}{r_{c} r^{c}}\left(g_{a b}-\frac{2 r_{a} r_{b}}{r_{c} r^{c}}\right), \quad k^{a b}=-r_{c} r^{c}\left(g^{a b}-\frac{2 r^{a} r^{b}}{r_{c} r^{c}}\right) .
$$

Then $k^{a b} h_{b c}=\delta_{c}^{a}$, and a straightforward calculation shows that Hamilton's equations on the future tube take the form

$$
\hbar \frac{\mathrm{d} x^{a}}{\mathrm{~d} s}=-k^{a b} \frac{\partial H}{\partial r^{b}} \quad \text { and } \quad \hbar \frac{\mathrm{d} r^{a}}{\mathrm{~d} s}=k^{a b} \frac{\partial H}{\partial x^{b}} .
$$

That the signs in (25) are reversed in comparison with (3) is an artefact of the convention that defines the future tube by points of the form $x^{a}-\mathrm{i} r^{a}$ with $r^{a}$ time-like and future-pointing. But what is not so obvious, and comes perhaps as a surprise, is that the quadratic form $h_{a b}$ is positive definite, thus defining a Riemannian metric on the future tube, given by

$$
\mathrm{d} s^{2}=h_{a b}\left(\mathrm{~d} x^{a} \mathrm{~d} x^{b}+\mathrm{d} r^{a} \mathrm{~d} r^{b}\right) .
$$

As a consequence we see that the arc-length along a smooth curve can be taken as a canonical parameterization of the phase-space trajectory. In particular, in situations where two or more particles are interacting, the phase space of the system as a whole can be taken to be the product of the phase spaces of the individual systems, with an overall positive definite metric, thus leading to a natural way of synchronizing the dynamics of the constituents.

\section{Relativistic phase-space geometry}

As a number of authors have pointed out, there are several distinct but ultimately equivalent ways of arriving at the geometrical structure of the future tube [31-41]. Building on these works, we pursue here an alternative approach to the geometry of $\Gamma$ that ties in naturally with quantum measurement theory. We begin with the Hilbert space $\mathcal{H}=L^{2}(\Gamma, \mathcal{O})$ of squareintegrable holomorphic functions on the future tube. If we let $f, g$ be elements of $\mathcal{H}$, then for their inner product we write

$$
\langle\bar{g} \mid f\rangle=\int_{\Gamma} f(z) \bar{g}(\bar{z}) \mathrm{d} \mu_{z}
$$

where

$$
\mathrm{d} \mu_{z}=\frac{1}{16} \mathrm{~d}^{4} z \mathrm{~d}^{4} \bar{z}
$$

denotes the usual Lebesgue measure on $\Gamma$. The fact that such functions constitute a Hilbert space is nontrivial, for it is not immediately obvious that any Cauchy sequence in $L^{2}(\Gamma, \mathcal{O})$ 
converges to an element of $L^{2}(\Gamma, \mathcal{O})$. That such convergence holds follows as a consequence of a well-known bound [42], which states that for any compact subset $Q \subset \Gamma$ there exists a constant $C_{Q}$ such that for all $\phi \in L^{2}(\Gamma, \mathcal{O})$ we have

$$
\sup _{z \in Q}|\phi(z)| \leqslant C_{Q}\|\phi\|
$$

where

$$
\|\phi\|=\left(\int_{\Gamma} \phi(z) \bar{\phi}(\bar{z}) \mathrm{d} \mu_{z}\right)^{1 / 2}
$$

Now let $\left\{\phi^{n}\right\}_{n \in \mathbb{N}}$ be an orthonormal basis for $\mathcal{H}$ so that

$$
\int_{\Gamma} \phi^{n}(z) \bar{\phi}_{m}(\bar{z}) \mathrm{d} \mu_{z}=\delta_{m}^{n}
$$

We introduce the Bergman kernel $[43,44]$ on $\Gamma$ by setting

$$
K(z, \bar{w})=\sum_{n} \bar{\phi}_{n}(\bar{w}) \phi^{n}(z)
$$

which is independent of the choice of orthonormal basis. Thus $K(z, \bar{w})$ is holomorphic in $z$ and antiholomorphic in $w$, and for any holomorphic function $f \in \mathcal{H}$ we evidently have

$$
\int_{\Gamma} K(z, \bar{w}) f(w) \mathrm{d} \mu_{w}=f(z) .
$$

We thus see that the Bergman kernel acts as an identity operator or reproducing kernel on $\mathcal{H}$. In particular, for all $x, y, z \in \Gamma$ we have the identity

$$
\int_{y} K(x, \bar{y}) K(y, \bar{z}) \mathrm{d} \mu_{y}=K(x, \bar{z})
$$

Now consider a smooth curve

$$
\gamma: \sigma \in[0,1] \mapsto w_{\sigma} \in \Gamma
$$

For each value of the parameter $\sigma$ the function $\psi_{\sigma}: \Gamma \rightarrow \mathbb{C}$ defined by

$$
\psi_{\sigma}(z)=K\left(z, \bar{w}_{\sigma}\right)
$$

is holomorphic and square integrable. It follows that $\psi_{\sigma}$ describes a curve in $\mathcal{H}$ as $\sigma$ varies, so we can work out the length along $\gamma$ by use of the Fubini-Study metric [25, 32]:

$$
\mathrm{d} s^{2}=\frac{\int \mathrm{d} \psi_{\sigma}(z) \mathrm{d} \bar{\psi}_{\sigma}(\bar{z}) \mathrm{d} \mu_{z}}{\int \psi_{\sigma}(z) \bar{\psi}_{\sigma}(\bar{z}) \mathrm{d} \mu_{z}}-\frac{\left|\int \mathrm{d} \psi_{\sigma}(z) \bar{\psi}_{\sigma}(\bar{z}) \mathrm{d} \mu_{z}\right|^{2}}{\left(\int \psi_{\sigma}(z) \bar{\psi}_{\sigma}(\bar{z}) \mathrm{d} \mu_{z}\right)^{2}}
$$

A calculation then shows that

$$
\mathrm{d} s^{2}=\frac{\partial^{2} \log K(w, \bar{w})}{\partial w^{a} \partial \bar{w}^{b}} \mathrm{~d} w^{a} \mathrm{~d} \bar{w}^{b} .
$$

Thus, the Fubini-Study metric on $\mathcal{H}$ induces a Kähler metric on $\Gamma$. This is the so-called Bergman metric [44]. The ideas involved with the Bergman kernel and the associated metric can be elucidated by considering the elementary example of a bounded domain $\mathbb{C}^{+}$of $\mathbb{C}$ 
defined by $\mathrm{i}(z-\bar{z})>0$. Writing $z=x-\mathrm{i}$, the domain can be expressed as the half plane for which $r>0$. Thus $\mathbb{C}^{+}$can be thought of as representing a one-dimensional future tube, i.e. a phase space in zero space dimensions, with only time and energy. An example of a set of orthonormal functions on $\mathbb{C}^{+}$is given by

$$
\phi^{n}(z)=2 \sqrt{\frac{n}{\pi}} \frac{(\mathrm{i}+z)^{n-1}}{(\mathrm{i}-z)^{n+1}},
$$

from which one can easily verify that the corresponding Bergman kernel takes the form

$$
K_{\mathbb{C}^{+}}(z, \bar{w})=\sum_{n=1}^{\infty} \phi^{n}(z) \bar{\phi}_{n}(\bar{w})=-\frac{1}{\pi(z-\bar{w})^{2}} .
$$

The associated Bergman metric is then the usual hyperbolic metric of the half plane. Note that under the map $w=f(z)$ given by the Cayley transform

$$
f(z)=\frac{\mathrm{i}+z}{\mathrm{i}-z}
$$

points of $\mathbb{C}^{+}$are mapped invertibly to points of the unit disk $|w|<1$, which is the so-called Poincaré disk $\mathbb{D}$. The orthonormal basis elements are then mapped to

$$
\phi_{n}(w)=\sqrt{\frac{n}{\pi}} w^{n-1},
$$

and a short calculation shows that the kernel function becomes

$$
K_{\mathbb{D}}(z, \bar{w})=\frac{1}{\pi(1-z \bar{w})^{2}} .
$$

In the case of the future tube, the Bergman kernel can be worked out explicitly, and we have

$$
K(z, \bar{w})=\left(\frac{2^{3} \cdot 4 !}{\pi^{4}}\right) \frac{1}{\left[g_{a b}\left(z^{a}-\bar{w}^{a}\right)\left(z^{b}-\bar{w}^{b}\right)\right]^{4}} .
$$

Note that similar to case of the half plane $\mathbb{C}^{+}$, the future tube can be mapped to a 'unit ball' domain $\mathbb{B}$, which acts as the higher-dimensional analogue of the Poincare disk $\mathbb{D}$. Specifically, if we write the points of $\Gamma$ in the $2 \times 2$ matrix form

$$
\hat{Z}=z^{0} \hat{I}+z^{1} \hat{\sigma}_{x}+z^{2} \hat{\sigma}_{y}+z^{3} \hat{\sigma}_{z}
$$

where $\hat{I}$ denotes the $2 \times 2$ identity matrix and $\hat{\sigma}_{x}, \hat{\sigma}_{y}, \hat{\sigma}_{z}$ are the Pauli matrices, the future tube is defined by the condition that

$$
\hat{I}-\hat{Z}^{\dagger} \hat{Z}>0
$$

where the inequality here means that the left side is a positive-definite matrix. The Cayley transform of the future tube in this representation is given by

$$
W=\mathrm{i}(\hat{I}-\hat{Z})(\hat{I}+\hat{Z})^{-1},
$$

which is the analogue of (41) for the half plane. With this transformation in mind, the numerical factor $V_{4}=\pi^{4} /\left(2^{3} \cdot 4\right.$ !) appearing in (44) can then be seen as arising from the Euclidean volume of the ball domain [45]. In the literature of the future tube, it is common to adopt 
the characterization in terms of $\mathbb{B}$, since this allows for a somewhat simpler treatment of the group-theoretic analysis associated with conformal transformations [31-37]. However, for in the consideration of quantum theory we find it more transparent to work directly with the standard characterization of the future tube as the domain $\Gamma$ in complexified Minkowski space. In particular, for its Bergman metric, substitution of (44) into (38) gives

$$
\frac{\partial^{2} \log K(z, \bar{z})}{\partial z^{a} \partial \bar{z}^{b}}=h_{a b}
$$

where $h_{a b}$ turns out to be none other than the metric (24) that we introduced earlier using the Kelvin transformation. Since a Bergman metric is fully determined by the complex analytic structure of the underlying domain, it follows that $h_{a b}$ admits the symmetry group of $\Gamma$, which is the 15-parameter conformal group of Minkowski space. These phase-space symmetries are generated by Hamiltonian flows on $\Gamma$.

\section{Quantum states}

Going forward, now let $u, v, w, x, y, z$ denote points of $\Gamma$. Having introduced the Hilbert space $\mathcal{H}$ of holomorphic functions on $\Gamma$, we are now in a position to build a quantum theory. A general state will be a density matrix $\rho(y, \bar{z}) \in L^{2}(\Gamma, \mathcal{O}) \times L^{2}(\Gamma, \overline{\mathcal{O}})$. For such a state, we require the following: (a) that $\rho(y, \bar{z})=\bar{\rho}(\bar{z}, y)$, (b) that $\rho(y, \bar{z})$ should be positive, that is to say

$$
\int \bar{\alpha}(\bar{y}) \rho(y, \bar{z}) \alpha(z) \mathrm{d} \mu_{y} \mathrm{~d} \mu_{z} \geqslant 0
$$

for $\alpha(z) \in L^{2}(\Gamma, \mathcal{O})$, and (c) that it should have unit trace,

$$
\int K(z, \bar{y}) \rho(y, \bar{z}) \mathrm{d} \mu_{y} \mathrm{~d} \mu_{z}=\int \rho(z, \bar{z}) \mathrm{d} \mu_{z}=1 .
$$

A state is then said to be pure if $\rho(y, \bar{z})=\xi(y) \bar{\xi}(\bar{z})$ for some holomorphic function $\xi \in L^{2}(\Gamma, \mathcal{O})$ with unit norm. We observe that for both pure and mixed states the 'diagonal' function $\rho(z, \bar{z})$ takes the form of a probability density on $\Gamma$. That a probability density function on phase space arises naturally in the present context is significant, since the construction of such densities in configuration-space models for relativistic quantum mechanics is known to be problematic.

The interpretation of a density matrix is that it represents the quantum state of a relativistic event. Such an event is accompanied by position and momentum data. The fact that wave functions are holomorphic then prohibits the possibility that they can be concentrated with arbitrarily high precision in a given region of phase space. This follows from the fundamental inequality (29). Many aspects of the theory can be understood as being analogous to the Bargmann-Segal construction in nonrelativistic quantum mechanics [46, 47].

\section{Space-time transformations}

It is natural to enquire how space-time transformations are represented in $\mathcal{H}$. That is, we are interested in constructing a unitary representation of the action of the Poincare group $\mathcal{P}(\Gamma)$ on $\Gamma$. Such transformations are of the form

$$
P: z^{a} \mapsto L_{b}^{a} z^{b}+B^{a}
$$


where $B^{a}$ is a real four vector and $L_{b}^{a}$ satisfies

$$
g_{a b} L_{c}^{a} L_{d}^{b}=g_{c d}
$$

A family of unitary operators $\left\{\hat{U}_{P}, P \in \mathcal{P}(\Gamma)\right\}$ generating Poincaré transformations through the action $\hat{U}_{P}: \mathcal{H} \rightarrow \mathcal{H}$ can then be seen to take the form

$$
U(x, \bar{y})=K\left(L_{b}^{a} x^{b}+B^{a}, \bar{y}^{a}\right),
$$

where $K(x, \bar{y})$ is the Bergman kernel (44). To see that the operator $\hat{U}$ thus defined is unitary, it suffices to show that $\hat{U} \hat{U}^{\dagger}=\hat{\mathbb{1}}$, where on account of (32) the identity operator $\hat{\mathbb{1}}$ here in the phase-space coordinate representation is given by the kernel function. Then by (53) we have

$$
\bar{U}(y, \bar{x})=K\left(y^{a}, L_{b}^{a} \bar{x}^{b}+B^{a}\right),
$$

and it follows from (33) and (52) that

$$
\begin{aligned}
\int U(x, \bar{y}) \bar{U}(y, \bar{z}) \mathrm{d} \mu_{y} & =\int K(L x+B, \bar{y}) K(y, L \bar{z}+B) \mathrm{d} \mu_{y} \\
& =K(L x+B, L \bar{z}+B) \\
& =\left(\frac{2^{3} \cdot 4 !}{\pi^{4}}\right) \frac{1}{\left[g_{a b}\left(L_{c}^{a} x^{c}-L_{c}^{a} \bar{z}^{c}\right)\left(L_{d}^{b} x^{d}-L_{d}^{b} \bar{z}^{d}\right)\right]^{4}} \\
& =K(x, \bar{z}),
\end{aligned}
$$

as desired. It is also apparent that $\hat{U}_{P^{\prime}} \hat{U}_{P}=\hat{U}_{P^{\prime} P}$ for all $P, P^{\prime} \in \mathcal{P}(\Gamma)$, so we conclude that (53) gives a unitary representation of the Poincaré group on the Hilbert space $\mathcal{H}=L^{2}(\Gamma, \mathcal{O})$.

More generally, a representation of the full 15-parameter conformal group can also be identified by use of the kernel function. To see this, we consider first the four-dimensional subgroup $\mathcal{S}(\Gamma)$ of special conformal transformations, given by

$$
S: z^{a} \mapsto \frac{z^{a}+z^{2} \lambda^{a}}{1+2 \lambda \cdot z+\lambda^{2} z^{2}}
$$

where $\lambda^{a}$ is a real four-vector, and we write $\lambda \cdot z=g_{a b} \lambda^{a} z^{b}, \lambda^{2}=g_{a b} \lambda^{a} \lambda^{b}$, and $z^{2}=g_{a b} z^{a} z^{b}$. The transformation (56) is obtained by applying an inversion $z^{a} \mapsto z^{a} /\left(g_{b c} z^{b} z^{c}\right)$, then shifting the result by $\lambda^{a}$, and finally applying a further inversion. The unitary operator $\hat{U}_{S}$ generating such a transformation on states in $\mathcal{H}$ is given by

$$
U(x, \bar{y})=\frac{1}{\left(1+2 \lambda \cdot x+\lambda^{2} x^{2}\right)^{4}} K\left(\frac{x^{a}+x^{2} \lambda^{a}}{1+2 \lambda \cdot x+\lambda^{2} x^{2}}, \bar{y}\right) .
$$

To see that $\hat{U}_{S}$ defines a unitary representation of the group $\mathcal{S}(\Gamma)$ on $\mathcal{H}$, we let $S \in \mathcal{S}(\Gamma)$ be parameterized by $\lambda^{a}$ and $S^{\prime} \in \mathcal{S}(\Gamma)$ be parameterized by $\mu^{a}$, and consider the action of $\hat{U}_{S^{\prime}} \hat{U}_{S}$ on a generic state $\psi(x) \in \mathcal{H}$. A calculation gives

$$
\hat{U}_{S^{\prime}} \hat{U}_{S} \psi(x)=\frac{1}{\left(1+2 \nu \cdot x+\nu^{2} x^{2}\right)^{4}} \psi\left(\frac{x^{a}+x^{2} \nu^{a}}{1+2 \nu \cdot x+\nu^{2} x^{2}}\right),
$$

where $\nu^{a}=\lambda^{a}+\mu^{a}$, and it follows that $\hat{U}_{S^{\prime}} \hat{U}_{S}=\hat{U}_{S^{\prime} S}$. The remaining component of the conformal group is the one-parameter dilatation group $\mathcal{D}(\Gamma)$, which consists of transformations 
of the form $D: z^{a} \mapsto \Lambda z^{a}$, where $\Lambda$ is a strictly positive real number. It should be apparent that the unitary operator generating a dilatation with parameter $\Lambda$ is

$$
U(x, \bar{y})=\Lambda^{4} K\left(\Lambda x^{a}, \bar{y}^{a}\right) .
$$

The corresponding action of a dilatation on a state $\psi(z) \in \mathcal{H}$ is thus $D: \psi(z) \mapsto \Lambda^{4} \psi(\Lambda z)$, and we see that (59) defines a unitary representation of the dilatation group on $L^{2}(\Gamma, \mathcal{O})$.

The idea that we exploit in arriving at explicit phase-space representations for unitary operators is the fact that on a Hilbert space with a reproducing kernel, every operator admits an integral representation [43]. Thus, for example, the generator of the space-time translation $z^{a} \rightarrow z^{a}+b^{a}$, where $b^{a}$ is a real four-vector, is given by $b^{a} \hat{P}_{a}$, where $\hat{P}_{a}=\mathrm{i} \hbar \partial / \partial x^{a}$ is the fourmomentum operator; but the four momentum operator $\hat{P}_{a}$ admits the following phase-space representation:

$$
P_{a}(z, \bar{w})=-\frac{3 \cdot 2^{9} \hbar \mathrm{i}}{\pi^{4}} \frac{g_{a b}\left(z^{b}-\bar{w}^{b}\right)}{\left[g_{a b}\left(z^{a}-\bar{w}^{a}\right)\left(z^{b}-\bar{w}^{b}\right)\right]^{5}} .
$$

\section{Quantum measurements}

To make sense of the notion of quantum detection in a relativistic setting we need positive operator-valued measures [48-50]. In the present context a POVM can be formed by taking a collection $\Phi$ of positive operators $\left\{\phi_{A}(y, \bar{z})\right\}_{A \in \mathcal{B}}$ on phase space labelled by elements of the Borel $\sigma$-algebra $\mathcal{B}(\Gamma)$ generated by the open subsets of $\Gamma$. We require $\Phi$ to have the following properties: (a) $\phi_{A}(y, \bar{z})$ is positive for each $A \in \mathcal{B}$, (b) $\phi_{\Gamma}(y, \bar{z})=K(y, \bar{z})$, and (c) for any countable collection of disjoint sets $\left\{A_{n}\right\}_{n \in \mathbb{N}}$ in $\mathcal{B}$ with union $A=\cup_{n \in \mathbb{N}} A_{n}$ it holds that

$$
\phi_{A}(x, \bar{z})=\sum_{n} \phi_{A_{n}}(x, \bar{z}) .
$$

We consider now a measurement operation appropriate for detecting the location of an event in phase space. The POVM is defined by

$$
\phi_{A}(x, \bar{z})=\int_{y \in A} K(x, \bar{y}) K(y, \bar{z}) \mathrm{d} \mu_{y}, \quad A \in \mathcal{B} .
$$

The recorded outcome of such a measurement will be a measurable set $A$ in phase space: for instance, the detection of a particle in a certain space-time region, accompanied by a fourmomentum taking values in a certain range. One can have in mind, for example, the detection of a cosmic ray. By (33) and (62), the probability that the event lies in the set $A \in \mathcal{B}$ is

$$
\mathbb{P}(A)=\int \phi_{A}(y, \bar{z}) \rho(z, \bar{y}) \mathrm{d} \mu_{y} \mathrm{~d} \mu_{z}=\int_{z \in A} \rho(z, \bar{z}) \mathrm{d} \mu_{z} .
$$

We see, in particular, in accordance with our earlier discussion, that $\rho(z, \bar{z})$ is the probability density for the outcome, and hence that the expectation value of any measurable function $F$ : $\Gamma \rightarrow \mathbb{R}$ is given by the integral

$$
\mathbb{E}[F]=\int F(z, \bar{z}) \rho(z, \bar{z}) \mathrm{d} \mu_{z},
$$

which is well-defined and finite providing that

$$
\int|F(z, \bar{z})| \rho(z, \bar{z}) \mathrm{d} \mu_{z}<\infty .
$$


Once a measurement has been performed and the outcome recorded, the state of the system changes. To model this we require a transformation operator of the Krauss type [51, 52]:

$$
T_{A}(u, v, \bar{x}, \bar{y})=\int_{w \in A} \frac{K(u, \bar{w}) K(v, \bar{w}) K(w, \bar{x}) K(w, \bar{y})}{K(w, \bar{w})} \mathrm{d} \mu_{w} .
$$

One can verify directly that the partial trace of the state transformation operator generates the POVM. That is, we have

$$
\int T_{A}(x, y, \bar{y}, \bar{z}) \mathrm{d} \mu_{y}=\phi_{A}(x, \bar{z})
$$

for each $A \in \mathcal{B}$. Now suppose that the system is initially in the state $\rho_{\text {in }}(y, \bar{v})$. Then after the measurement we find that

$$
\rho_{\text {out }}(u, \bar{x})=\frac{\int T_{A}(u, v, \bar{x}, \bar{y}) \rho_{\text {in }}(y, \bar{v}) \mathrm{d} \mu_{v} \mathrm{~d} \mu_{y}}{\int T_{A}(z, v, \bar{z}, \bar{y}) \rho_{\text {in }}(y, \bar{v}) \mathrm{d} \mu_{v} \mathrm{~d} \mu_{y} \mathrm{~d} \mu_{z}},
$$

which represents the transformed state that results when the measurement determines that the phase-space event lies in the set $A \in \mathcal{B}$. Substituting (66) in (68), and making use of the reproducing property (33), we deduce that

$$
\rho_{\text {out }}(u, \bar{x})=\frac{\int_{z \in A} \Psi_{z}(u, \bar{x}) \rho_{\text {in }}(z, \bar{z}) \mathrm{d} \mu_{z}}{\int_{z \in A} \rho_{\text {in }}(z, \bar{z}) \mathrm{d} \mu_{z}},
$$

where

$$
\Psi_{z}(u, \bar{x})=\frac{K(u, \bar{z}) K(z, \bar{x})}{K(z, \bar{z})}
$$

is the density matrix associated with the normalized wave function

$$
\psi_{z}(u)=\frac{K(u, \bar{z})}{[K(z, \bar{z})]^{1 / 2}} .
$$

Then in the limit that the recorded outcome shrinks to a phase-space point, we find that

$$
\rho_{\text {out }}(u, \bar{x})=\Psi_{z}(u, \bar{x}) .
$$

In what follows we shall refer to a pure state of the form (71) as a coherent state with focus $z$. We observe, in particular, that by virtue of (44) we have

$$
\psi_{z}(u)=\frac{8 \sqrt{3}}{\pi^{2}} \frac{\left[g_{a b}\left(z^{a}-\bar{z}^{a}\right)\left(z^{b}-\bar{z}^{b}\right)\right]^{2}}{\left[g_{a b}\left(u^{a}-\bar{z}^{a}\right)\left(u^{b}-\bar{z}^{b}\right)\right]^{4}} .
$$

We can also refer to a pure density matrix of the form (70) as a coherent state, and we note that the family of such density matrices satisfies a completeness relation of the form

$$
\int_{z} \Psi_{z}(x, \bar{y}) K(z, \bar{z}) \mathrm{d} \mu_{z}=K(x, \bar{y}) .
$$

That such a relation should hold is characteristic of the properties of coherent states [53, 54] and follows from the fundamental identity (34), as does the structure of the POVM given by (62). It is interesting then to note that the coherent states arising in the present context are in 
one-to-one correspondence with the so-called 'elementary states' that arise in the theory of zero rest mass fields [9].

The foregoing analysis shows that when the measurement apparatus detects that an event has taken place in a region $A$ of phase space, the output state will in general be a mixed state, given by the weighted average of the coherent state $\Psi_{z}(u, \bar{x})$ over $z \in A$ with respect to the renormalized density

$$
\rho_{A}(z, \bar{z})=\frac{\rho_{\text {in }}(z, \bar{z})}{\int_{y \in A} \rho_{\text {in }}(y, \bar{y}) \mathrm{d} \mu_{y}} .
$$

If, however, the record shows a specific phase-space point $z$ as the result, then the output density matrix will be the coherent state $\Psi_{z}(u, \bar{x})$ parameterized by $z$. At the other extreme, if the measurement is performed but the outcome is not recorded, then the focus is smeared over the whole of the phase space, representing a decoherence effect, and we obtain

$$
\rho_{\text {out }}(u, \bar{x})=\int \Psi_{z}(u, \bar{x}) \rho_{\text {in }}(z, \bar{z}) \mathrm{d} \mu_{z} .
$$

\section{Properties of coherent states}

On the matter of the interpretation of the coherent states, we remark that the family of coherent states $\left\{\psi_{z}(u)\right\}$ parameterized by $z \in \Gamma$ is Poincaré invariant in the sense that under the unitary transformation (53) one has

$$
\hat{U} \psi_{z}(u)=\psi_{w}(u)
$$

where $w^{a}=L_{b}^{a} z^{b}-B^{a}$. In other words, the action of the unitary representation of the Poincaré transformation on a coherent state $\psi_{z}(u)$ focussed at $z \in \Gamma$ is the coherent state $\psi_{w}(u)$ focussed at $w$, where $w$ is the result of the corresponding inverse Poincaré transformation on $z$.

More generally, one can show that manifold of coherent states is invariant under the action of the 15-parameter conformal group. To see this, consider first the dilatation group. From (59) we see that $\hat{U} \psi_{z}(u)=\psi_{w}(u)$, where $w^{a}=\Lambda^{-1} z^{a}$. Thus, the action of the dilatation on a coherent state $\psi_{z}(u)$ focussed at the phase-space point $z$ is a coherent state $\psi_{w}(u)$ focussed at $w$, where $w$ is the result of the corresponding inverse dilatation on $z$.

The action of the special conformal transformations on a coherent state is a little more subtle. Writing $\hat{U}_{\lambda}$ for the unitary operator (57), we find that

$$
\begin{aligned}
\hat{U}_{\lambda} \psi_{z}(u)= & \frac{8 \sqrt{3}}{\pi^{2}} \frac{[(z-\bar{z}) \cdot(z-\bar{z})]^{2}}{\left(1+2 \lambda \cdot u+\lambda^{2} u^{2}\right)^{4}}\left[\left(\frac{u+u^{2} \lambda}{1+2 \lambda \cdot u+\lambda^{2} u^{2}}-\bar{z}\right)\right. \\
& \left.\cdot\left(\frac{u+u^{2} \lambda}{1+2 \lambda \cdot u+\lambda^{2} u^{2}}-\bar{z}\right)\right]^{-4}
\end{aligned}
$$

A calculation then gives

$$
\begin{aligned}
& \left(\frac{u+u^{2} \lambda}{1+2 \lambda \cdot u+\lambda^{2} u^{2}}-\bar{z}\right) \cdot\left(\frac{u+u^{2} \lambda}{1+2 \lambda \cdot u+\lambda^{2} u^{2}}-\bar{z}\right) \\
& \quad=\frac{1-2 \lambda \cdot \bar{z}+\lambda^{2} \bar{z}^{2}}{1+2 \lambda \cdot u+\lambda^{2} u^{2}}[(u-\bar{w}) \cdot(u-\bar{w})],
\end{aligned}
$$


where

$$
w^{a}=\frac{z^{a}-z^{2} \lambda^{a}}{1-2 \lambda \cdot z+\lambda^{2} z^{2}} .
$$

Thus the terms involving $\left(1+2 \lambda \cdot u+\lambda^{2} u^{2}\right)$ appearing in (78) cancel, and we obtain

$$
\hat{U}_{\lambda} \psi_{z}(u)=\frac{8 \sqrt{3}}{\pi^{2}} \frac{[(z-\bar{z}) \cdot(z-\bar{z})]^{2}}{\left(1-2 \lambda \cdot \bar{z}+\lambda^{2} \bar{z}^{2}\right)^{4}} \frac{1}{[(u-\bar{w}) \cdot(u-\bar{w})]^{4}} .
$$

In fact, the right side of (81) is a phase-shifted version of the coherent state $\psi_{w}(u)$ centred at the phase-space point $w$ :

$$
\hat{U}_{\lambda} \psi_{z}(u)=\mathrm{e}^{\mathrm{i} \theta} \frac{8 \sqrt{3}}{\pi^{2}} \frac{[(w-\bar{w}) \cdot(w-\bar{w})]^{2}}{[(u-\bar{w}) \cdot(u-\bar{w})]^{4}},
$$

where the phase shift $\theta$ is given by

$$
\theta=\frac{1}{2 \mathrm{i}} \log \left[\frac{1-2 \lambda \cdot z+\lambda^{2} z^{2}}{1-2 \lambda \cdot \bar{z}+\lambda^{2} \bar{z}^{2}}\right]=\frac{1}{2 \mathrm{i}} \log \left[\frac{z^{2} \bar{w}^{2}}{\bar{z}^{2} w^{2}}\right]
$$

In other words, the result of the action of a special conformal transformation on a coherent state $\psi_{z}(u)$ focussed at $z$ is a coherent state $\psi_{w}(u)$ focussed at $w$ with a phase shift $\theta$, where $w$ is the result of the action of the corresponding inverse special conformal transformation on $z$. Since the physical state of a system is defined up to an overall phase (or, equivalently, the phase factor drops out if we consider the action of a conformal transformation on a pure-state density matrix), we deduce that the manifold of coherent states is invariant under the 15-parameter conformal group.

In calculations, it turns out to be convenient in many situations to work with Fourier transforms. In fact, there are some remarkable identities that turn out to be useful in this connection. The Fourier transform of an element $\psi \in L^{2}(\Gamma, \mathcal{O})$ is defined by

$$
\Psi\left(p^{a}\right)=\int_{\Gamma} \exp \left(\mathrm{i} p_{a} \bar{z}^{a}\right) \psi\left(z^{a}\right) \mathrm{d} \mu_{z} .
$$

The inverse Fourier transform is then given by

$$
\psi\left(z^{a}\right)=\frac{1}{8 \pi^{5}} \int_{V^{+}} \exp \left(-\mathrm{i} p_{a} z^{a}\right)\left(p_{b} p^{b}\right)^{2} \Psi\left(p^{a}\right) \mathrm{d}^{4} p
$$

where the integration is over the interior of the forward cone defined by

$$
V^{+}=\left\{p^{a}: p_{a} p^{a}>0, p^{0}>0\right\}
$$

The argument for (85) can be sketched as follows. Let $I\left(z^{a}\right)$ denote the outcome of the integral appearing on the right-hand side of (85). If we substitute (84) into the formula for $I\left(z^{a}\right)$, we get

$$
I\left(z^{a}\right)=\frac{1}{8 \pi^{5}} \int_{V^{+}} \exp \left(-\mathrm{i} p_{a} z^{a}\right)\left(p_{b} p^{b}\right)^{2} \int_{\Gamma} \exp \left(\mathrm{i} p_{a} \bar{w}^{a}\right) \psi\left(w^{a}\right) \mathrm{d} \mu_{w} \mathrm{~d}^{4} p .
$$

Then, reversing the order of integration, we have

$$
I\left(z^{a}\right)=\frac{1}{8 \pi^{5}} \int_{\Gamma}\left[\int_{V^{+}} \exp \left(-\mathrm{i} p_{a} z^{a}\right)\left(p_{b} p^{b}\right)^{2} \exp \left(\mathrm{i} p_{a} \bar{w}^{a}\right) \mathrm{d}^{4} p\right] \psi\left(w^{a}\right) \mathrm{d} \mu_{w} .
$$


Now, the inner integral can be carried out explicitly, and we obtain

$$
\frac{1}{8 \pi^{5}} \int_{V^{+}} \exp \left(-\mathrm{i} p_{a} z^{a}\right)\left(p_{b} p^{b}\right)^{2} \exp \left(\mathrm{i} p_{a} \bar{w}^{a}\right) \mathrm{d}^{4} p=K(z, \bar{w}),
$$

where the Bergman kernel is defined as in (44). An application of the reproducing property (33) then shows that $I\left(z^{a}\right)=\psi\left(z^{a}\right)$, and thus we obtain (85), the Fourier inversion formula.

Alternatively, suppose that we are given a map $\Psi: V^{+} \rightarrow \mathbb{C}$ on the positive cone such that

$$
\int_{V^{+}}\left(p_{b} p^{b}\right)^{2}\left|\Psi\left(p^{a}\right)\right|^{2} \mathrm{~d}^{4} p<\infty
$$

and we define a holomorphic function $\psi: \Gamma \rightarrow \mathbb{C}$ by use of (85). Let $J\left(p^{a}\right)$ denote the outcome of the integral appearing on the right side of (84). Then for $J\left(p^{a}\right)$ we obtain

$$
\begin{aligned}
J\left(p^{a}\right) & =\frac{1}{8 \pi^{5}} \int_{\Gamma} \exp \left(\mathrm{i} p_{a} \bar{z}^{a}\right) \int_{V^{+}} \exp \left(-\mathrm{i} q_{a} z^{a}\right)\left(q_{b} q^{b}\right)^{2} \Psi\left(q^{a}\right) \mathrm{d}^{4} q \mathrm{~d} \mu_{z} \\
& =\frac{1}{8 \pi^{5}} \int_{V^{+}}\left[\int_{\Gamma} \exp \left(\mathrm{i} p_{a} \bar{z}^{a}-\mathrm{i} q_{a} z^{a}\right) \mathrm{d} \mu_{z}\right]\left(q_{b} q^{b}\right)^{2} \Psi\left(q^{a}\right) \mathrm{d}^{4} q .
\end{aligned}
$$

Let us consider the inner integration first. Writing $z^{a}=x^{a}-\mathrm{i} r^{a}$ with $x^{a}$ real, and with $r^{a}$ timelike and future pointing, we have

$$
\mathrm{i} p_{a} \bar{z}^{a}-\mathrm{i} q_{a} z^{a}=\mathrm{i}\left(p_{a}-q_{a}\right) x^{a}-\left(p_{a}+q_{a}\right) r^{a} .
$$

Since $\mathrm{d} \mu_{u}=\mathrm{d}^{4} x \mathrm{~d}^{4} r$, we find that the $x$-integration over Minkowski space gives a delta function. Thus setting $\xi_{a}=p_{a}+q_{a}$ we have

$$
\int_{\Gamma} \exp \left(\mathrm{i} p_{a} \bar{z}^{a}-\mathrm{i} q_{a} z^{a}\right) \mathrm{d} \mu_{z}=(2 \pi)^{4} \delta^{4}\left(p^{a}-q^{a}\right) \int_{V^{+}} \exp \left(-\xi_{a} r^{a}\right) \mathrm{d}^{4} r .
$$

For the $r$-integration, we can pass to spherical coordinates. Then if we set $\xi^{2}=\left(\xi^{1}\right)^{2}+\left(\xi^{2}\right)^{2}+$ $\left(\xi^{3}\right)^{2}$ and $R^{2}=\left(r^{1}\right)^{2}+\left(r^{2}\right)^{2}+\left(r^{3}\right)^{2}$, because $r^{a}$ is time-like we find

$$
\begin{gathered}
\int_{V^{+}} \exp \left(-\xi_{a} r^{a}\right) \mathrm{d}^{4} r=\int_{r^{0}=0}^{\infty} \int_{R=0}^{r^{0}} \int_{\theta=0}^{\pi} \int_{\phi=0}^{2 \pi} \exp \left(-\xi_{0} r^{0}+\xi R \cos \theta\right) \\
\times R^{2} \mathrm{~d} r^{0} \mathrm{~d} R \sin \theta \mathrm{d} \theta \mathrm{d} \phi .
\end{gathered}
$$

Because $r^{a}$ is future pointing, the integrals can now be performed explicitly to give

$$
\int_{V^{+}} \exp \left(-\xi_{a} r^{a}\right) \mathrm{d}^{4} r=\frac{8 \pi}{\left(\xi_{a} \xi^{a}\right)^{2}}
$$

from which it follows that

$$
\int_{\Gamma} \exp \left(\mathrm{i} p_{a} \bar{z}^{a}-\mathrm{i} q_{a} z^{a}\right) \mathrm{d} \mu_{z}=\frac{8 \pi^{5}}{\left(q_{a} q^{a}\right)^{2}} \delta^{4}\left(p^{a}-q^{a}\right) .
$$

Inserting this expression back into (91) we immediately see that the result of the integral is given by $\Psi\left(p^{a}\right)$. 
By a similar argument it follows from (89) that if $\Psi(p)$ is the Fourier transform of a squareintegrable holomorphic function $\psi(z)$, and $\Phi(p)$ is the Fourier transform of a square-integrable holomorphic function $\phi(z)$, then we have a Parseval identity of the form

$$
\int_{\Gamma} \psi\left(z^{a}\right) \bar{\phi}\left(\bar{z}^{a}\right) \mathrm{d} \mu_{z}=\frac{1}{8 \pi^{5}} \int_{V^{+}} \Psi(p)\left(p_{a} p^{a}\right)^{2} \bar{\Phi}(p) \mathrm{d}^{4} p .
$$

In the case of a coherent state $\psi_{z}(u)$ with focus $z \in \Gamma$, a calculation shows that its Fourier transform is given by

$$
\Psi_{z}\left(p^{a}\right)=\frac{8 \sqrt{3}}{\pi^{2}}\left[g_{a b}\left(z^{a}-\bar{z}^{a}\right)\left(z^{b}-\bar{z}^{b}\right)\right]^{2} \exp \left(\mathrm{i} \bar{z}_{a} p^{a}\right) .
$$

Now writing $z^{a}=x^{a}-\mathrm{i} r^{a}$, we have $z^{a}-\bar{z}^{a}=-2 \mathrm{i} r^{a}$, so

$$
\Psi_{z}\left(p^{a}\right)=\frac{2^{7} \sqrt{3}}{\pi^{2}}\left(r_{a} r^{a}\right)^{2} \mathrm{e}^{-r_{a} p^{a}} \mathrm{e}^{\mathrm{i} x_{a} p^{a}}
$$

This relation shows that as $z^{a}$ varies the Fourier component $\Psi_{z}\left(p^{a}\right)$ behaves like a plane wave in Minkowski space that has been extended into the future tube, but is damped exponentially for large $r^{a}$. We notice, in particular, that when $r^{a}$ is large, corresponding to the case where focal point lies in a low-mass region of $\Gamma$, the damping of the high-energy Fourier components is significant.

\section{Phase-space localization}

With a view to getting a better understanding of the degree of localization in phase space that might be achievable in such a detection experiment, let us consider properties of the coherent states in more detail.

For each choice of the focal point $z \in \Gamma$, the associated coherent state is represented by the normalized wave function $\psi_{z}(u)$. Now, if $|\phi\rangle \in L^{2}(\mathcal{H}, \mathcal{O})$ is any other normalized state, we have the Cauchy-Schwartz inequality

$$
\left\langle\psi_{z} \mid \phi\right\rangle\left\langle\phi \mid \psi_{z}\right\rangle \leqslant 1 \text {. }
$$

It then follows immediately from (33), (71) and (100) that

$$
\phi(z) \bar{\phi}(\bar{z}) \leqslant K(z, \bar{z})
$$

for all $z \in \Gamma$, which shows that the amplitude of any pure state at a phase-space point $z$ is bounded by the amplitude at $z$ of the coherent state with focus $z$.

Hence the coherent states are the most sharply peaked states, and the peak of a coherent state occurs at its focus. It thus makes sense that if the measurement outcome takes the form of a specific point in phase space, then the transformed state should be peaked as much as possible at that point, and hence a coherent state with that peak, as we obtained in (72).

An interesting physical interpretation of the inequality (101) can be deduced if we write $\operatorname{Im}\left(z^{a}\right)=-r^{a}$ and make use of the phase-space correspondence (21). It follows from (44) that

$$
K(z, \bar{z})=\frac{3}{4 \pi^{4} \hbar^{8}}\left(g_{a b} p^{a} p^{b}\right)^{4}
$$

Then if we let

$$
M_{z}=\left(g_{a b} p^{a} p^{b}\right)^{1 / 2}
$$


denote the mass associated with the phase-space point $z^{a}$, we can write (101) in the form of a localization bound on the probability density. In particular, we obtain

$$
\rho(z, \bar{z}) \leqslant \frac{3}{4 \pi^{4} \hbar^{8}} M_{z}^{8}
$$

which shows that states cannot be localized very sharply in regions of phase space with low mass, but that for higher mass a much greater degree of localization can be achieved.

We can thus think of (104) as a localization theorem for relativistic quantum theory. Suppose that a phase-space event of a relativistic system is characterized by a pure state $\phi(z)$. Then the probability of detecting the event is determined by the normalized density function

$$
\rho(z, \bar{z})=\phi(z) \bar{\phi}(\bar{z})
$$

The localization theorem shows that the maximum value that the density function can take, at any given point in the eight-dimensional phase space, is

$$
\rho_{\max }(z, \bar{z})=\frac{3}{4 \pi^{4} \lambda_{z}^{8}},
$$

where $\lambda_{z}$ denotes the reduced Compton wavelength associated with the phase-space point $z$. Now, if an event is detected to have occurred at a specific phase-space point $z$, then the resulting output wave function will be given by the corresponding coherent state, for which the bound in (104) is saturated. Since the density function $\rho(z, \bar{z})$ has to integrate to unity over the phase space, one is thus able to conclude that for a system of short Compton wavelength the event will be highly localized in phase space. It follows that we can view the coherent states as representing in some sense the most 'classical' type of state that can be formed over the relativistic phase space.

To gain further intuition about the nature of localization, let us consider an example in which the state of the system is given by a holomorphic wave function $|\xi\rangle$ which we take to be normalized, and an experimentalist wishes to determine whether a localized event at a phase space point $z$ can be detected. Since this is a 'yes-no' type of question, a projective measurement is appropriate, and accordingly we consider the projection operator

$$
\hat{\Pi}_{z}=\left|\psi_{z}\right\rangle\left\langle\psi_{z}\right|
$$

where $\left|\psi_{z}\right\rangle$ denotes a normalized coherent state focussed at the point $z$. Here as an aid to intuition we introduce the usual bra-ket notation and we add 'hats' to operators. Then $|\xi\rangle$ can be split uniquely into a part that is localized at $z$ and a part that is orthogonal to $\left|\psi_{z}\right\rangle$, so

$$
|\xi\rangle=\left|\psi_{z}\right\rangle\left\langle\psi_{z} \mid \xi\right\rangle+\left(\hat{\mathbb{1}}-\hat{\Pi}_{z}\right)|\xi\rangle
$$

In fact, it is not difficult to show that if a holomorphic function $\theta(u) \in L^{2}(\mathcal{H}, \mathcal{O})$ is orthogonal to a coherent state $\psi_{z}(u)$ with focal point $z$, then $\theta(u)$ vanishes at the focal point. Thus, we can say in a meaningful sense that any state that is orthogonal to a coherent sate with focus $z$ is delocalized away from the focal point.

If the outcome of the projective measurement is affirmative, then, by the usual Lüders-type rules for projective measurements [55], the transformed state will be the localized state $\psi_{z}(u)$. Otherwise, we obtain the delocalized wave function given by the uniquely determined relative state. In particular, if the initial state is given by the holomorphic function $\xi(u)$ then the outcome 
of a projective measurement based on the projection operator associated with the coherent state $\left|\psi_{z}\right\rangle$ will be affirmative with probability

$$
p=\left\langle\psi_{z} \mid \xi\right\rangle\left\langle\xi \mid \psi_{z}\right\rangle=\frac{\xi(z) \bar{\xi}(\bar{z})}{K(z, \bar{z})} .
$$

Now suppose that the wave function $|\xi\rangle$ is itself a coherent state, centred at the phase-space point $w=x-\mathrm{i} r$. Then we have $|\xi\rangle=\left|\psi_{w}\right\rangle$, and the probability $p$ of obtaining a 'yes' outcome in a projective measurement involving the projection operator $\hat{\Pi}_{z}$ with $z=x^{\prime}-\mathrm{i} r^{\prime}$ is given by $\left|\left\langle\psi_{w} \mid \psi_{z}\right\rangle\right|^{2}$. A calculation shows that

$$
p=\left[\frac{(w-\bar{w}) \cdot(w-\bar{w})(z-\bar{z}) \cdot(z-\bar{z})}{(w-\bar{z}) \cdot(w-\bar{z})(z-\bar{w}) \cdot(z-\bar{w})}\right]^{4}
$$

which can be viewed as a cross ratio between the four points $w^{a}, z^{a}, \bar{w}^{a}$ and $\bar{z}^{a}$. In fact, it is well known that the transition probability between two pure states in nonrelativistic quantum mechanics admits a natural geometric interpretation as a cross ratio between points in complex projective space [29].

But in the present context what is surprising is that the cross ratio involves points in complex Minkowski space. It is a straightforward exercise to verify that the cross ratio is conformally invariant and hence a fortiori Poincaré invariant.

\section{Discussion}

In summary, we have shown that the future tube possesses a phase-space geometry appropriate both for (a) formulation of a consistent Hamiltonian mechanics for relativistic systems, and (b) construction of a quantum theory of space-time events. In particular, the Hilbert space of square-integrable holomorphic functions on the future tube can be interpreted as the pure state space of relativistic quantum mechanics. The resulting structure is rich enough to allow for the development of a manifestly covariant theory of measurement for the detection of phase-space events. The theory incorporates a natural transformation rule for the quantum state after the measurement, a concept that has hitherto been lacking in relativistic quantum theory. We are also able to gain some understanding of the extent to which relativistic events can be localized. An upper bound for the phase-space probability density can be determined, which is inversely proportional to the eighth power of the Compton wavelength. The upper bound is achieved at any given point in phase space by the probability density associated with the phase-space coherent state that has its focal point at that point.

\section{Acknowledgments}

DCB acknowledges support from the Russian Science Foundation, Grant 20-11-20226. We thank M Hoban for helpful discussions.

\section{Data availability statement}

No new data were created or analysed in this study. 


\section{ORCID iDs}

Dorje C Brody (D) https://orcid.org/0000-0002-9242-9554

Lane P Hughston (D) https://orcid.org/0000-0003-1185-934X

\section{References}

[1] Dirac P A M 1958 The theory of gravitation in Hamiltonian form Proc. R. Soc. A 246 333-43

[2] Wightman A S 1956 Quantum field theory in terms of vacuum expectation values Phys. Rev. 101 860-6

[3] Wightman A 1960 Quantum field theory and analytic functions of several complex variables $J$. Indian Math. Soc. 24 625-77

[4] Araki H 1961 Wightman functions, retarded functions and their analytic continuations Prog. Theor Phys. Suppl. 18 83-125

[5] Streater R F and Wightman A S 1964 PCT, Spin and Statistics, and All That (New York: Benjamin)

[6] Schweber S S 1962 An Introduction to Relativistic Quantum Field Theory (New York: Harper and Row)

[7] Penrose R 1967 Twistor algebra J. Math. Phys. 8 345-66

[8] Penrose R 1967 Twistor quantization and curved space-time Int. J. Theor. Phys. 1 61-99

[9] Penrose R and MacCallum M A H 1972 Twistor theory: an approach to the quantization of fields and space-time Phys. Rep. 16 241-316

[10] Mackey G W 1963 The Mathematical Foundations of Quantum Mechanics (New York: Benjamin)

[11] Landau L and Peierls R 1931 Erweiterung des unbestimmtheitsprinzips für die relativistische quantentheorie Z. Phys. $6956-69$

[12] Aharonov Y and Albert D Z 1981 Can we make sense out of the measurement process in relativistic quantum mechanics? Phys. Rev. D 24 359-70

[13] Marolf D and Rovelli C 2002 Relativistic quantum measurement Phys. Rev. D 66023510

[14] Peres A and Terno D R 2004 Quantum information and relativity theory Rev. Mod. Phys. 76 93-123

[15] Nikolov P A and Todorov I T 1982 Space-time versus phase-space approach to relativistic particle dynamics Twistor Geometry and Non-Linear Systems ed H D Doebner and T D Palev (Berlin: Springer)

[16] Marsden J E, Montgomery R, Morrison P J and Thompson W B 1986 Covariant Poisson brackets for classical fields Ann. Phys., NY $16929-47$

[17] Sorge H, Stöcker H and Greiner W 1989 Poincaré invariant Hamiltonian dynamics: modelling multihadronic interactions in a phase space approach Ann. Phys., NY 192 266-306

[18] Woodhouse N M J 1992 Geometric Quantization 2nd edn (Oxford: Oxford University Press)

[19] Currie D G 1963 Interaction contra classical relativistic Hamiltonian particle mechanics J. Math. Phys. 4 1470-88

[20] Kaiser G 1977 Phase-space approach to relativistic quantum mechanics. I. Coherent-state representation for massive scalar particles J. Math. Phys. 18 952-9

[21] Komar A 1978 Constraint formalism of classical mechanics Phys. Rev. D 18 1881-6

[22] Rohrlich F 1979 Relativistic Hamiltonian dynamics I. Classical mechanics Ann. Phys., NY 117 $292-322$

[23] King M J and Rohrlich F 1980 Relativistic Hamiltonian dynamics. II. Momentum-dependent interactions, confinement and quantization Ann. Phys., NY 130 350-94

[24] Kibble T W B 1979 Geometrization of quantum mechanics Commun. Math. Phys. 65 189-201

[25] Page D N 1987 Geometrical description of Berry's phase Phys. Rev. A 363479

[26] Gibbons G W 1992 Typical states and density matrices J. Geom. Phys. 8 147-62

[27] Hughston L P 1995 Geometric aspects of quantum mechanics Twistor Theory ed S A Huggett Lecture Notes in Pure and Applied Mathematics vol 169 (New York: Marcel Dekker) pp 59-79

[28] Ashtekar A and Schilling T A 1998 Geometrical formulation of quantum mechanics On Einstein's Path ed A Harvey (New York: Springer) pp 23-65

[29] Brody D C and Hughston L P 2001 Geometric quantum mechanics J. Geom. Phys. 38 19-53

[30] Bengtsson I and Zyczkowski K 2006 Geometry of Quantum States (Cambridge: Cambridge University Press)

[31] Uhlmann A 1963 Remark on the future tube Acta Phys. Pol. 24293

[32] Kobayashi S and Nomizu K 1969 Foundations of Differential Geometry vol 2 (New York: Wiley) 
[33] Uhlmann A 1972 Some properties of the future tube (Karl Marx Universität report KMU-HEP 7029) pp $1-14$

[34] Rühl W 1972 Distributions on Minkowski space and their connection with analytic representations of the conformal group Commun. Math. Phys. 27 53-86

[35] Rühl W 1973 Field representations of the conformal group with continuous mass spectrum Commun. Math. Phys. 30 287-302

[36] Odzijéwicz A 1976 A model of conformal kinematics Int. J. Theor. Phys. 15 575-93

[37] Carey A L 1977 Induced representations, reproducing kernels and the conformal group Commun. Math. Phys. 52 77-101

[38] Coquereaux R and Jadczyk A 1990 Conformal theories, curved phase spaces, relativistic wavelets and the geometry of complex domains Rev. Math. Phys. 2 1-44

[39] Odzijéwicz A 1992 Coherent states and geometric quantization Commun. Math. Phys. 150 385-413

[40] Segreev A G and Vladimirov V S 1994 Complex analysis in the future tube Several Complex Variables II ed G M Khenkin and A G Vitushkin (New York: Springer)

[41] Gibbons G W 2000 Holography and the future tube Class. Quantum Grav. 17 1071-9

[42] Krantz S G 1982 Function Theory of Several Complex Variables (New York: Wiley)

[43] Meschkowski H 1962 Hilbertsche Räume mit Kernfunktion (Berlin: Springer)

[44] Bergman S 1970 The Kernel Function and Conformal Mapping (Providence, RI: American Mathematical Society)

[45] Hua L K 1963 Harmonic Analysis of Functions of Several Complex Variables in the Classical Domains (Providence, RI: American Mathematical Society)

[46] Bargmann V 1961 On a Hilbert space of analytic functions and an associated integral transform part I Commun. Pure Appl. Math. 14 187-214

[47] Segal I E 1963 Mathematical Problems of Relativistic Physics (Providence, RI: American Mathematical Society)

[48] Davies E B and Lewis J T 1970 An operational approach to quantum probability Commun. Math. Phys. 17 239-60

[49] Davies E B 1976 Quantum Theory of Open Systems (London: Academic)

[50] Holevo A S 1982 Probabilistic and Statistical Aspects of Quantum Theory (Amsterdam: NorthHolland)

[51] Kraus K 1971 General state changes in quantum theory Ann. Phys., NY 64 311-35

[52] Krauss K 1983 States, Effects, and Operations (Berlin: Springer)

[53] Peremolov A M 1972 Coherent states for arbitrary Lie group Commun. Math. Phys. 26 222-36

[54] Ali S T, Antoine J P and Gazeau J P 2014 Coherent States, Wavelets, and their Generalizations (New York: Springer)

[55] Lüders G 1951 Über die zustandsänderung durch den meßprozeß Ann. Phys. 8 322-8 